\title{
Digital spotlighting filtering optimization for SAR imaging
}

\author{
Eric J. Balster ${ }^{*}$ (D), David B. Mundy², Andrew M. Kordik² and Kerry L. Hill ${ }^{3}$
}

\begin{abstract}
In this paper, a synthetic aperture radar (SAR) image formation simulator is used to objectively evaluate the parameter selection within the digital spotlighting process. Specifically, recommendations for the filter type and filter order of the low-pass filters used in the range and azimuth decimation processes within the digital spotlighting algorithm are determined to maximize image quality and minimize computational cost. Results show that a finite impulse response low-pass filter with a Taylor $(\bar{n}=5)$ window applied provides the highest image quality over a wide range of filter orders and decimation factors. Additionally, a linear relationship between filter length and decimation factor is found.
\end{abstract}

Keywords: Spotlight SAR simulator, Digital spotlighting, Filter order, Filter windowing

\section{Introduction}

Of the several methods for synthetic aperture radar (SAR) image formation, back-projection has many attractive attributes. A SAR image may be iteratively formed by back-projecting each pulse return independently and using a simple summation of the back-projected returns to form the SAR image. Thus, each pulse return may be processed in parallel. Additionally, the back-projection process automatically ortho-rectifies the image allowing for ease of geo-location of the processed imagery. However, there is a significant computational cost to backprojection. Although it is a conceptually straightforward algorithm, it carries a computational complexity of $O\left(N^{3}\right)$ which does not scale.

A common way to combat the non-scalable nature of the back-projection algorithm is to break the SAR phase history into manageable pieces and back-project different locations of the SAR image independently. This process, called digital spotlighting [1], lends itself to further parallel processing and scalability of the back-projection algorithm.

Several methods in the literature have used digital spotlighting in SAR image formation. In [2], a new algorithm

*Correspondence: ebalster1@udayton.edu

'Department of Electrical and Computer Engineering, Kettering Laboratory, University of Dayton, Rm. 341, 300 College Park, Dayton, OH 45469, USA

Full list of author information is available at the end of the article for circular SAR imaging is developed using digital spotlighting for acceleration. Additionally, in [3], digital spotlighting is used to enhance the back-projection process in terms of reduced Doppler aliasing. In [4], digital spotlighting is used to processes wide bandwidth and wide beamwidth P-3 SAR data.

However, many of the methods provided in the literature do not give guidance on several of the parameters involved in the digital spotlighting process, namely filter order and filter type. The choice of filter and the length of its impulse response play a large role in the formed SAR image quality and the computational complexity of the digital spotlighting algorithm.

This paper presents a series of tests to determine filter design guidelines for digital spotlighting in the SAR image formation process. A SAR image formation simulator is used which generates synthetic SAR video phase history (VPH) from digital imagery $[5,6]$. The synthetic VPH data is then formed into SAR imagery via digital spotlighting, inverse Fourier transform (iFFT) processing, and back-projection. Because the synthetic VPH is generated from digital imagery, the quality of the formed SAR imagery is able to be objectively evaluated with wellknown image processing metrics. In this study, structured similarity (SSIM) is used to provide simple guidelines on filter design in SAR digital spotlighting [7].

Test results show that a finite impulse response (FIR) filter Taylor window $(\bar{n}=5)$ provides the highest image 


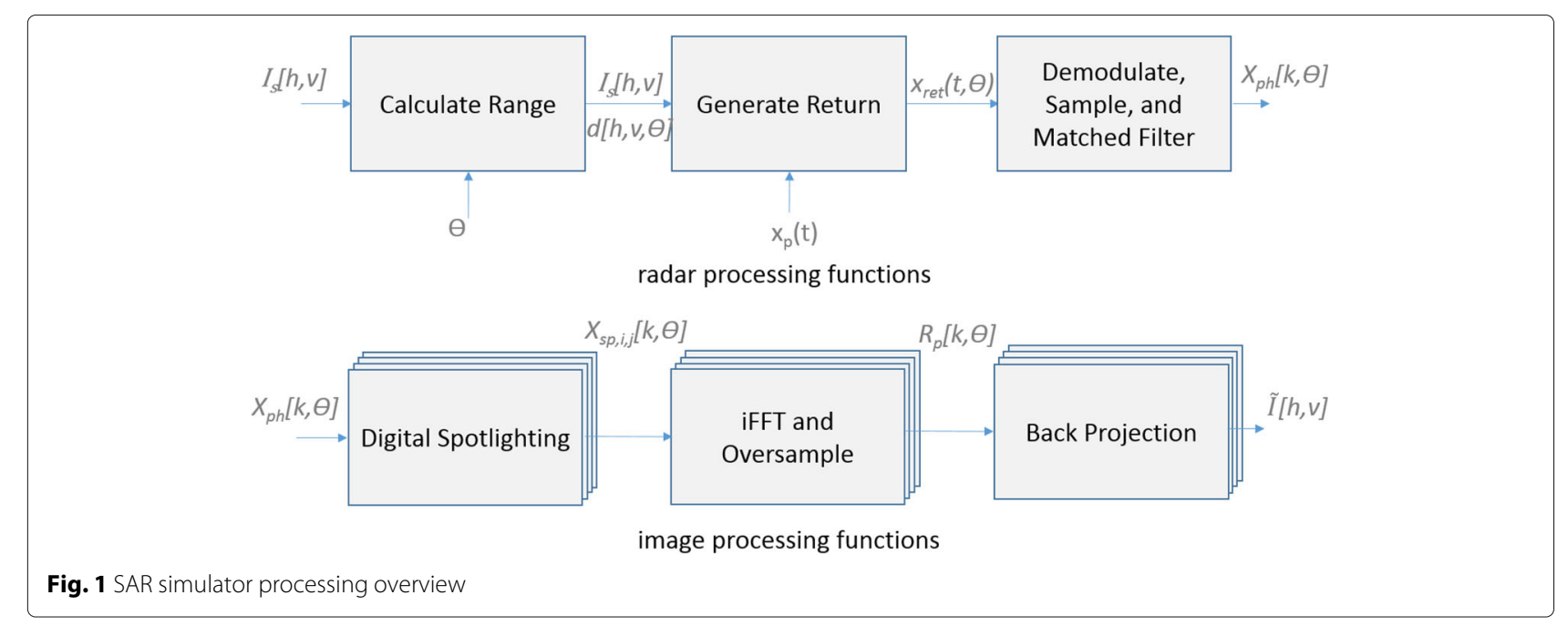

quality over several windowing methods tested, and a simple linear relationship between the number of digitally spotlit segments $D$ and filter order $M$ is discovered.

Following the introduction, Section 2 provides an overview of the SAR simulator for image formation. Section 3 provides a detailed overview of the digital spotlighting algorithm. Section 4 shows the results of FIR filter window evaluation, and Section 5 provides an evaluation of the filter order. Section 6 provides some concluding remarks.

\section{SAR processing overview}

In order to objectively evaluate different parameters of the digital spotlighting processing chain, a spotlight SAR simulator is used to generate synthetic VPH from digital imagery. Then, traditional SAR processing methods are used to generate imagery from the synthetic VPH data. Because the synthetic VPH is generated from imagery, traditional image processing metrics may be used to objectively evaluate the processing methods. Figure 1 gives a block diagram of the SAR simulator process.

As shown in Fig. 1, the SAR simulator is comprised of six processing steps. The first set of processing steps in the simulator form the VPH from a digital image $I[\cdot]$. This set of functions are referred to as the radar processing functions. The Calculate Range function determines the range from the beginning of the imaging patch to each pixel in the image, $d[h, v, \theta]$, where $\theta$ is the angular position of the radar with respect to the image. The Generate Return function sums up delayed and scaled linear frequency modulated (LFM) pulses to form the return SAR signal, given by:

$$
x_{r e t}(t, \theta)=\sum_{i, j} I_{s}[i, j] x_{p}\left(t-\frac{2 d[i, j, \theta]}{c}\right) \text {, }
$$
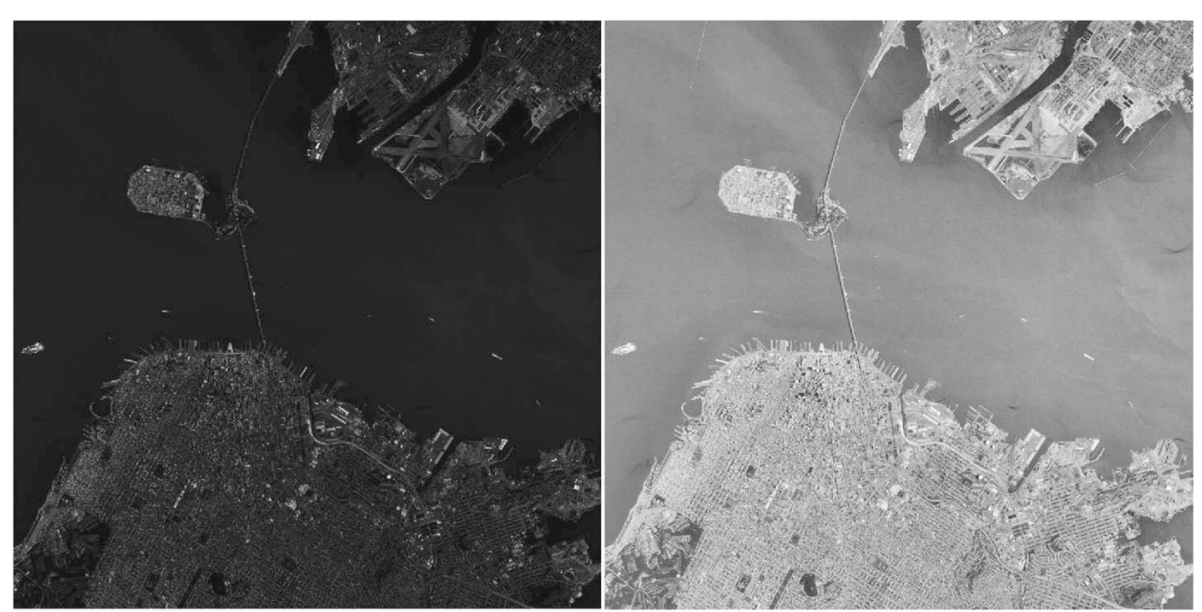

Fig. 2 Scaling imagery. Left: $I_{s}[\cdot], b=10$. Right: $\frac{\left.R_{d B}-20 \log _{10}\left(l_{s}[\cdot]\right]\right)}{R_{d B}}$ 
where $x_{p}(t)$ is the emitted radar pulse, and $I_{s}[\cdot]$ is the digital image $I[\cdot]$ scaled in magnitude.

The Demodulate, Sample, and Matched filter function is a set of standard SAR processing techniques to generate the VPH from $x_{\text {ret }}(t, \theta)$. The synthetic VPH is labeled as $X_{\mathrm{ph}}[k, \theta]$ in Fig. 1 .

The next set of functions are referred to as image processing functions. These functions take the synthetic VPH data and form SAR imagery. The first image processing function is the digital spotlighting function. This function allows segmentation of the VPH data so that different sections of the imaging scene may be formed independently. The next process is the iFFT and Oversample function which utilizes the inverse fast Fourier Transform to convert the VPH data into a range profile, $R_{p}[k, \theta]$. The final image processing function is the back-projection function which paints the range profile over the imaging scene, provides phase error correction, and sums up each back-projected pulse return to form the SAR image.

Each of the processing functions given in Fig. 1 is developed in detail in [8], with the exception of digital spotlighting, which is detailed in Section 3.

\subsection{SAR dynamic range}

Generally, SAR imagery is displayed in decibels due to its large dynamic range. Typical imagery, however, has a relatively limited dynamic range. Thus, to effectively utilize imagery in creating synthetic phase history, the dynamic range of the imagery is stretched. First, we can determine the dynamic range of the radar by determining the number of bits in the A/D process.

$$
R_{d B}=20 \log _{10}\left(2^{-b}\right)
$$

where $R_{d B}$ is the resolution of the $\mathrm{A} / \mathrm{D}$ converter in decibels, and $b$ is the number of bits resolved in the A/D converter. Consider we have an image $I[\cdot]$, where $I[h, v]$, where $h$ is the horizontal pixel index (column), and $v$ is the vertical index (row). To stretch the dynamic range of imagery, we have:

$$
I_{S}[h, v]=10^{(\max (I[\cdot])-I[h, v]) \frac{R_{d B}}{20}},
$$

where $I_{s}[\cdot]$ is the stretched image. If the original image, $I[\cdot]$, has a range of $[0,1]$, the scaled imagery $I_{s}[\cdot]$ also ranges from $[0,1]$. However, its dynamic range is shifted to match that of the A/D converter. Figure 2 gives an

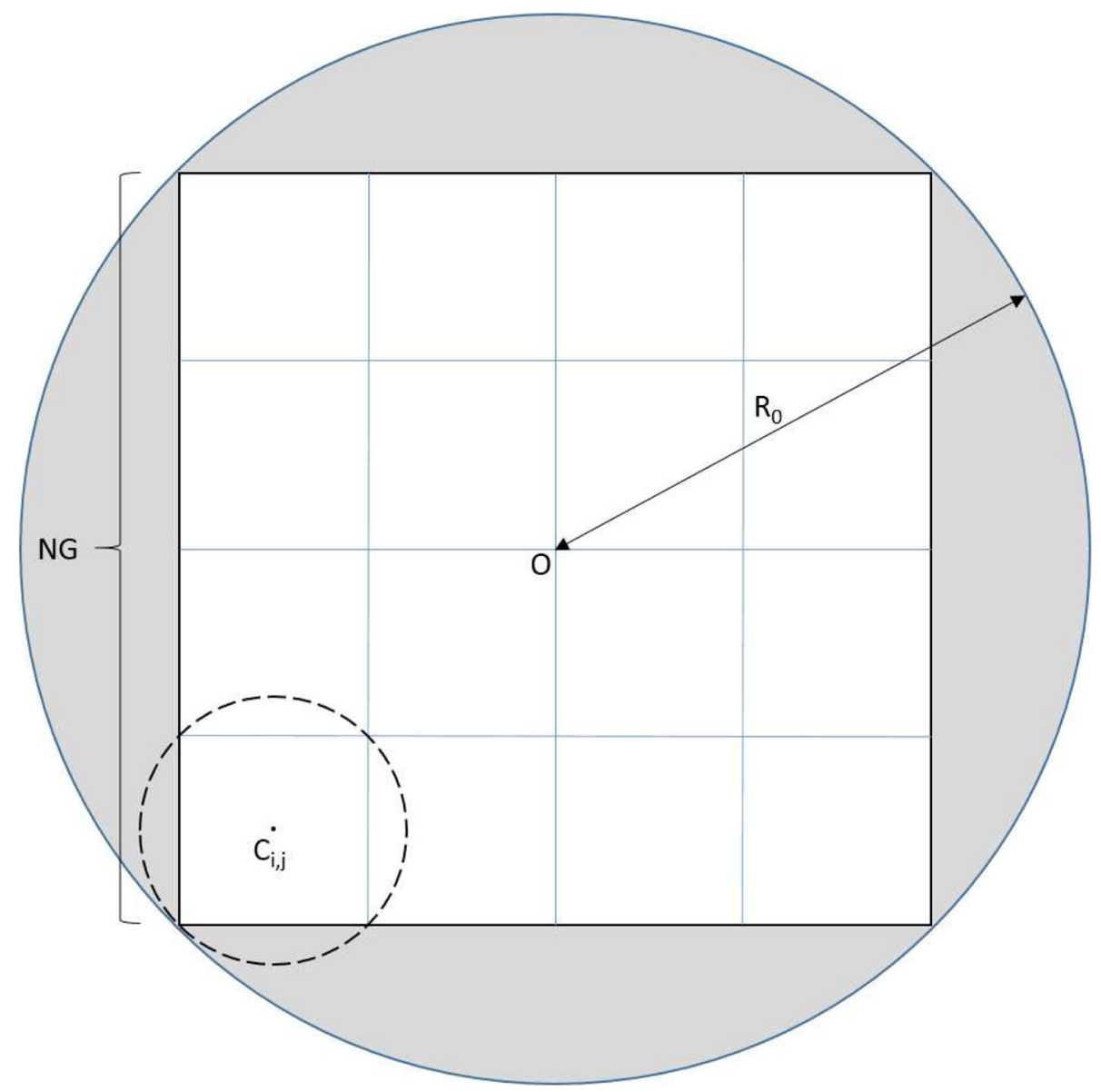

Fig. 3 Imaging regions. $D=4,(i, j)=(3,0)$ 
example of the "San Francisco" image scaled to $b=10$ (i.e., $R_{d B}=-60.21$ ).

As shown in Fig. 2, the scaled imagery is then best visualized in decibels.

\section{Digital spotlighting method}

Assume a scene with alias-free imaging region of radius $R_{0}$. From that scene, a SAR image of $(N G) \times(N G)$ meters may be formed, where $N$ is the number of row/column pixels, and $G$ is the ground sample distance.

$$
N G=\sqrt{2} R_{0}
$$

Both $N$ and $G$ are determined by:

$$
G=\frac{c}{2 B W_{p}}
$$

and

$$
N=\frac{c}{2 \sqrt{2} G f_{\Delta}}
$$

where $B W_{p}$ is the LFM pulse bandwidth, $f_{\Delta}=\frac{1}{T_{r}}$ is the frequency step size, $T_{r}$ is the duration of the return signal, and $c$ is the speed of light. A depiction of the imaging region is given in Fig. 3.

One of the uses of digital spotlighting is to mitigate the computational burden of back-projection of the SAR VPH to image the scene. Since back-projection is an $O\left(N^{3}\right)$ algorithm, it does not scale, so digital spotlighting is a way to break up the phase history into computationally manageable sections for imaging via back-projection.

Given the imaging scene in Fig. 3, the original imaging canvas is $N \times N$ pixels. Through digital spotlighting, $D^{2}$ digitally spotlit segments are back-projected to form $\left\lceil\frac{N}{D}\right\rceil \times\left\lceil\frac{N}{D}\right\rceil$ pixel images. First, the central position of each of the the digitally spotlit scenes, given by $C_{i, j}$, is determined. The digitally spotlit scenes are indexed by $i$ and $j$ row and column indices (i.e., $i, j \in[0, D-1]$ ). The center

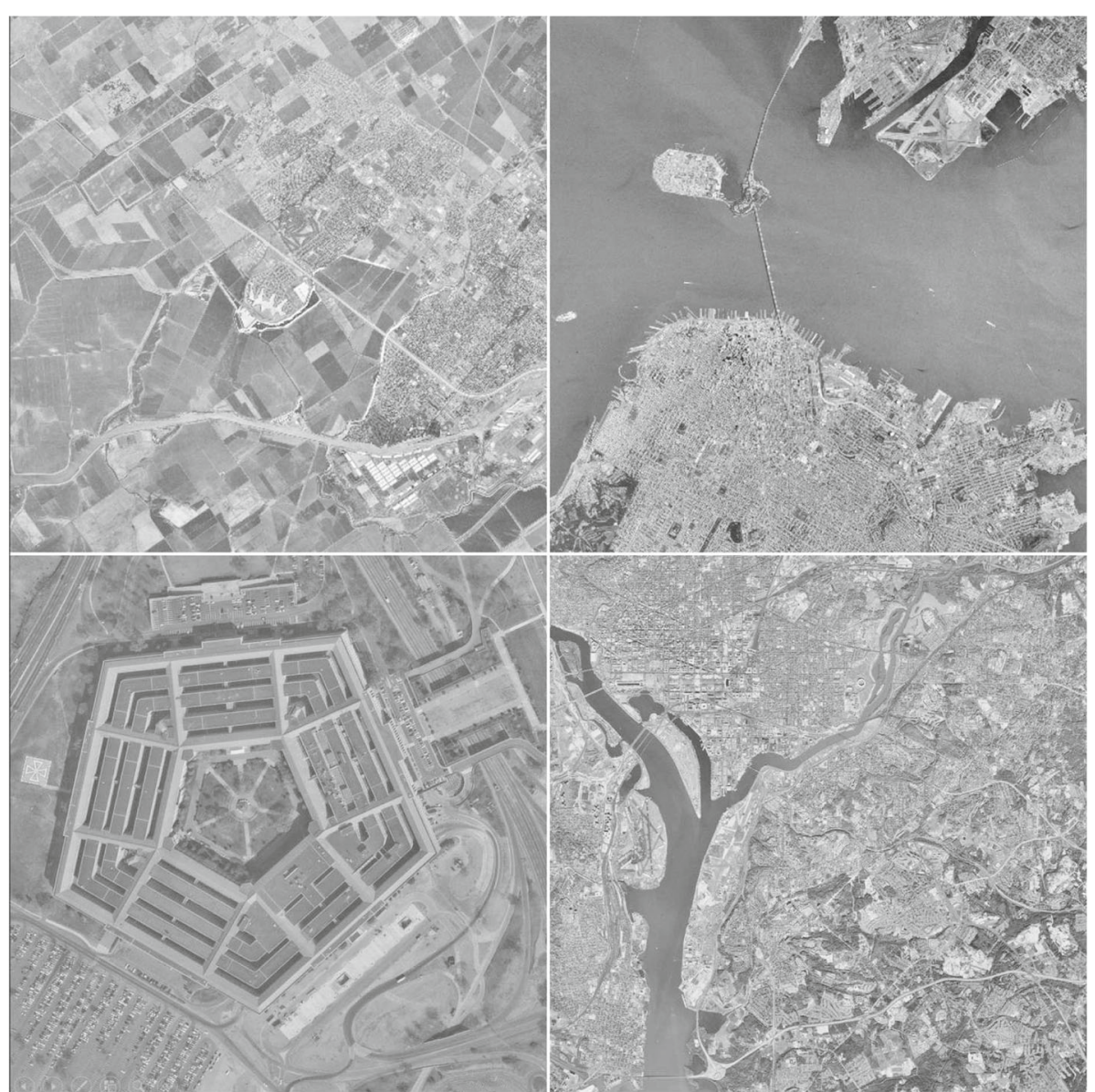

Fig. 4 Images used in testing. Top left: "Stockton." Top right: "San Francisco." Bottom left: "Pentagon." Bottom right: "Washington, D. C." N = 1024 for all images 


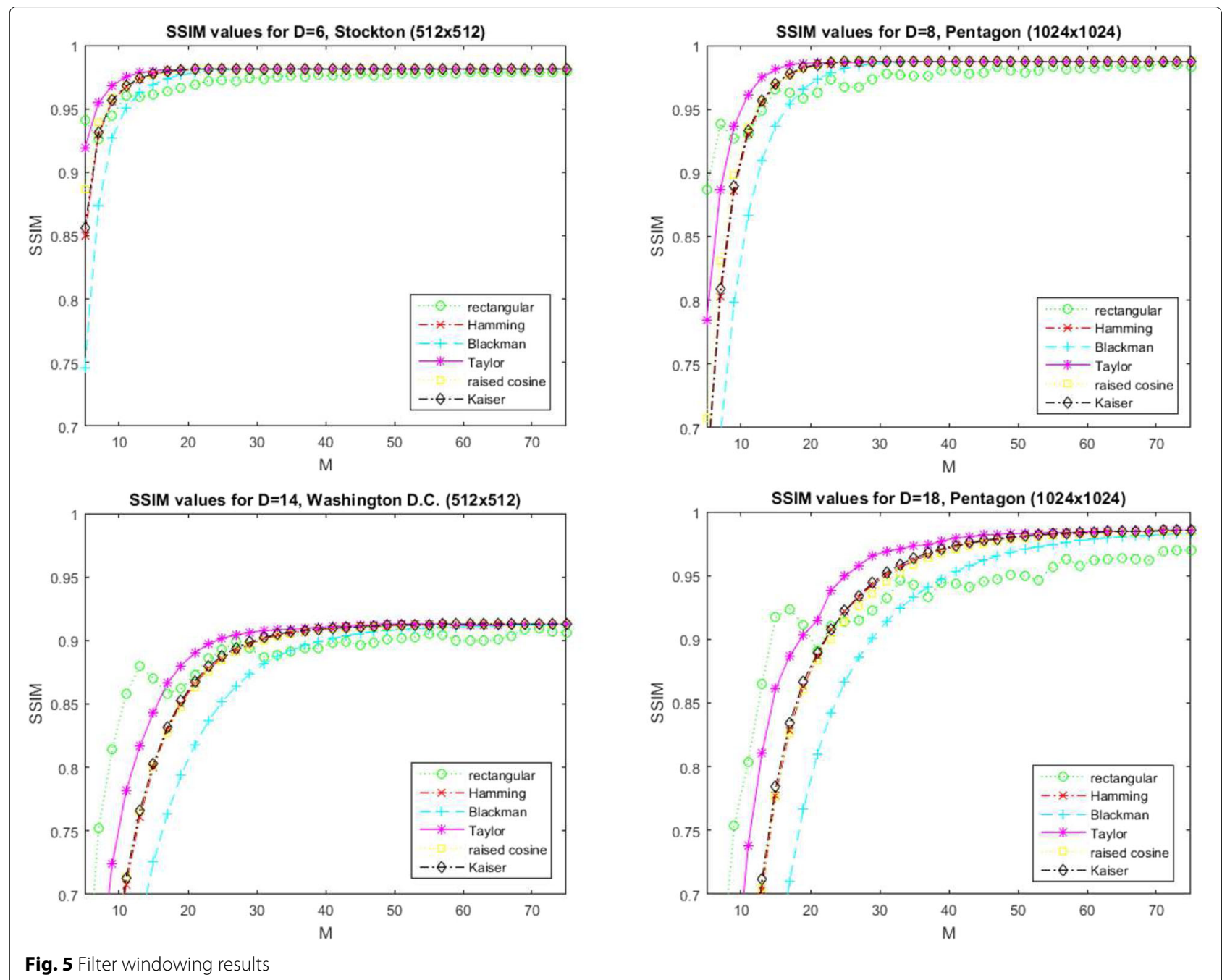

Fig. 5 Filter windowing results

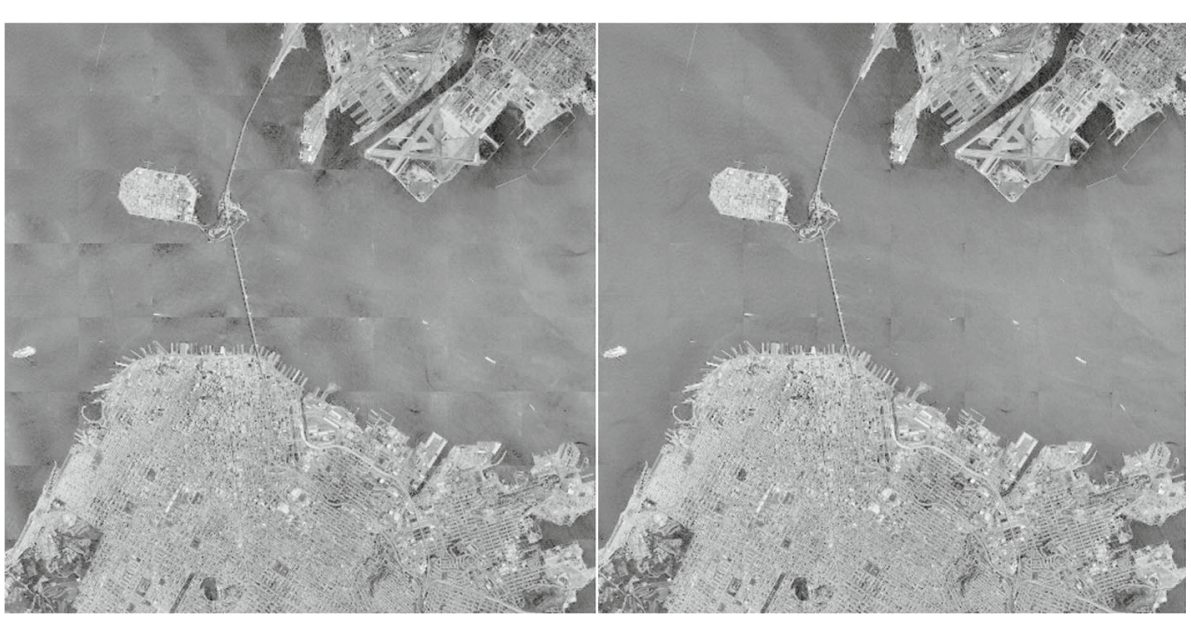

Fig. 6 Digitally spotlight "San Francisco" images. $D=8, M=19$. Left: rectangular window, $\mathrm{SSIM}=0.9304$. Right: Taylor $(\bar{n}=5)$ window, SSIM $=$ 0.9824 


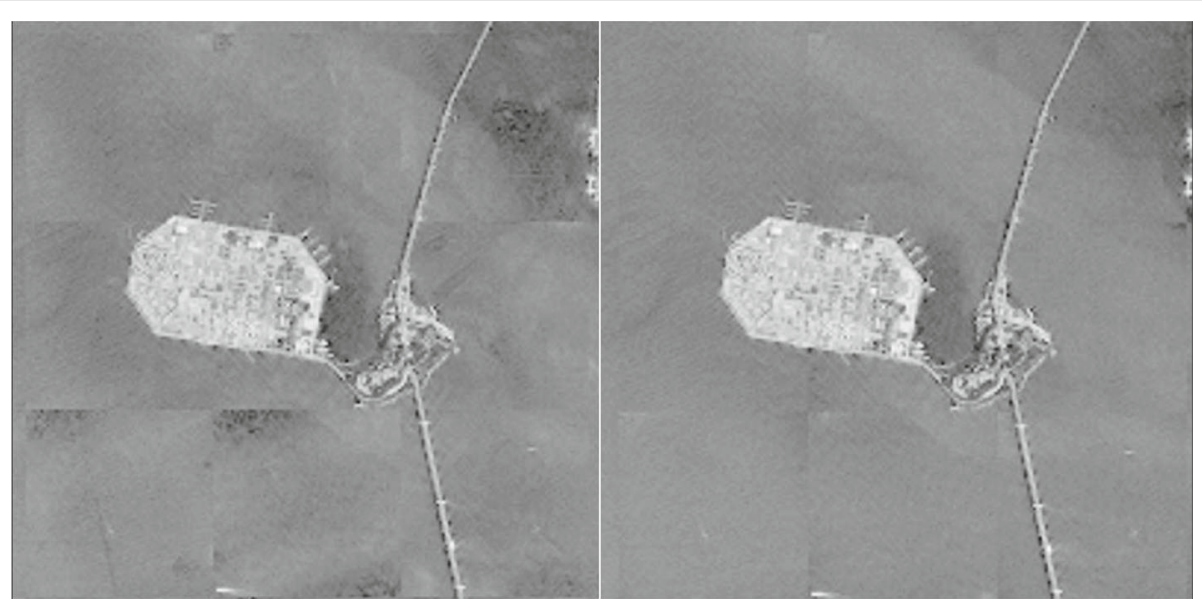

Fig. 7 Zoomed in portion of Fig. 6

of the digitally spotlit scene $(i, j)$ is given by:

$$
\begin{aligned}
& h_{C}(i, j)=\frac{2 j+1}{2}\left\lceil\frac{N}{D}\right\rceil \\
& v_{C}(i, j)=\frac{2 i+1}{2}\left\lceil\frac{N}{D}\right\rceil
\end{aligned}
$$

The pixel locations are converted to distances to the center of the imaging scene (scene center), indicated by $O$ in Fig. 3, by:

$$
\begin{aligned}
& d_{h}(i, j)=\left(h_{C}(i, j)-\frac{N}{2}\right) G \\
& d_{v}(i, j)=\left(\frac{N}{2}-v_{C}(i, j)\right) G
\end{aligned} .
$$

Digital spotlighting consists of four major processing steps:

- Repositioning of the antenna

- Re-centering of the phase history data

- Decimation in the range dimension

- Decimation in the azimuth dimension

\subsection{Repositioning of the antenna}

For each pulse return of the phase history, the position of the antenna must be updated to reflect the position of the new scene center. Assume we have an antenna position of $P=(X, Y, Z)$, where $X, Y$, and $Z$ are the latitude, longitude, and altitude, respectively, of the antenna, in meters. The new antenna position is, then, given by:

$$
P_{C}(i, j)=\left(R_{x}(i, j), R_{y}(i, j), R_{z}\right),
$$

where

$$
\begin{aligned}
& R_{x}(i, j)=X-X_{O}+d_{h}(i, j), \\
& R_{y}(i, j)=Y-Y_{O}+d_{\nu}(i, j), \\
& R_{z}=Z-Z_{O},
\end{aligned}
$$

and $P_{O}=\left(X_{O}, Y_{O}, Z_{O}\right)$ is the position of scene center $O$. When back-projecting the digitally spotlit area, $P_{C}$ is used for all distance calculations.
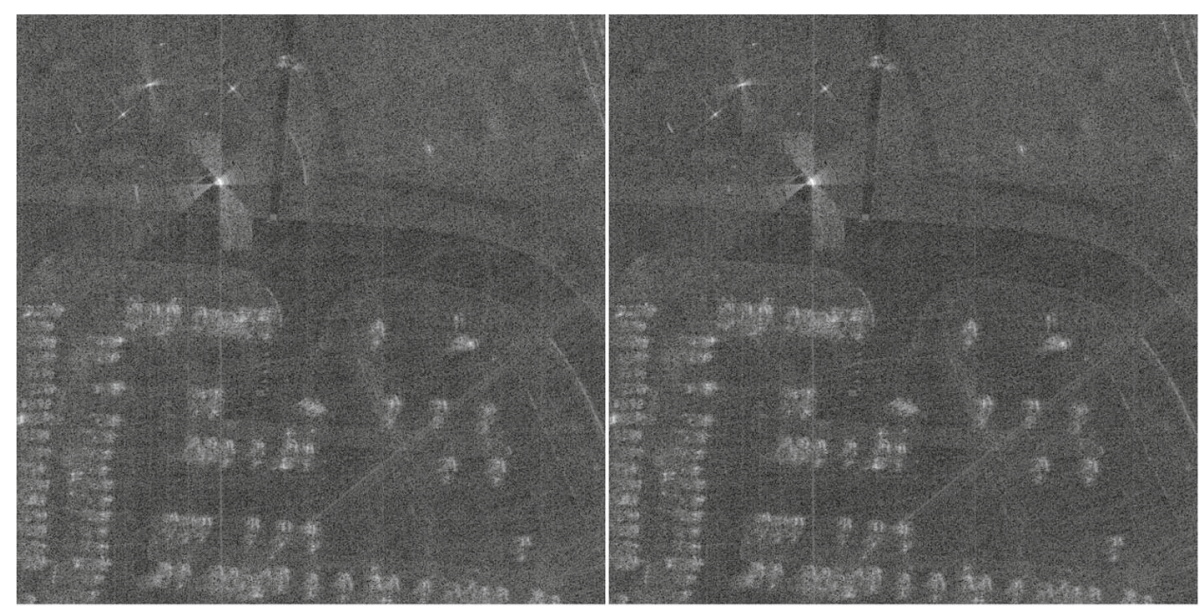

Fig. 8 Digitally spotlight "GOTCHA" images. $D=10, M=25$. Left: rectangular window. Right: Taylor $(\bar{n}=5)$ window 


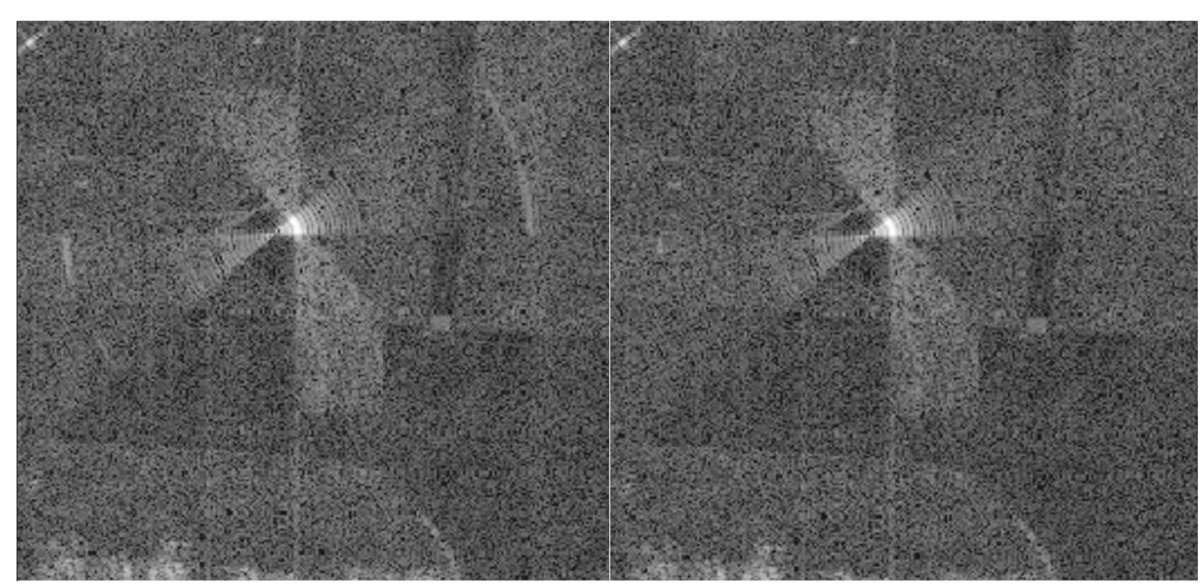

Fig. 9 Zoomed in portion of Figure 8

\subsection{Re-centering of the phase history data}

Re-centering of the phase history data applies a phase shift in order to place the digitally spotlit scene at zero frequency. Because the phase history is frequency dependent upon range, the differential range from the center of the spotlit scene to scene center is first calculated.

$$
\begin{aligned}
& R=\sqrt{\left(X-X_{O}\right)^{2}+\left(Y-Y_{O}\right)^{2}+\left(Z-Z_{O}\right)^{2}} \\
& R_{C}(i, j)=\sqrt{R_{x}(i, j)^{2}+R_{y}(i, j)^{2}+R_{z}^{2}} \\
& d_{R}(i, j)=R_{C}(i, j)-R
\end{aligned} .
$$

The re-centered phase history is then given by:

$$
X_{C, i, j}[k, \theta]=X_{p h}[k, \theta] e^{-\frac{j 4 \pi f[k] d_{R}(i, j)}{c}},
$$

where

$$
f[k]=f_{c}-\frac{B W_{p}}{2}+f_{\Delta} k .
$$

$X_{p h}[\cdot]$ is the original phase history data of the scene, and $X_{C, i, j}[\cdot]$ is the re-centered phase history about position $C_{i, j} . f[k]$ are the frequency bins in range, where $k \in[0, K)$, and $K$ is the number of range samples in $X_{p h}[\cdot] . f_{c}$ is the center frequency of the radar's LFM pulse.

\subsection{Decimation in the range dimension}

The re-centered data is then low-pass filtered with a discrete cutoff frequency of $\frac{\pi}{D}$ radians per sample, in which we obtain:

$$
X_{r l f, i, j}[k, \theta]=F_{r}\left\{X_{C, i, j}[k, \theta]\right\},
$$

where $F_{r}\{\cdot\}$ is the low-pass filtering operation in the range dimension. Then, the low-pass-filtered data may be downsampled by a factor of $D$.

$$
X_{r d, i, j}[k, \theta]=X_{r l f, i, j}[D k, \theta] .
$$

$X_{r d, i, j}[\cdot]$ is the phase history decimated in range for spotlit area $(i, j)$.

\subsection{Decimation in the azimuth dimension}

The alias-free azimuth resolution is used to determine the amount of decimation in the cross-range. The new azimuth step size is given by:

$$
\theta_{n \Delta}=\frac{c D}{4 \cos (\phi) R_{0}\left(f_{c}+\frac{B W_{p}}{2}\right)},
$$

where $\phi$ is the minimum elevation angle of the radar to the spotlit scene, given by:

$$
\phi=\arctan \frac{R_{z}}{\max \sqrt{R_{x}(i, j)^{2}+R_{y}(i, j)^{2}}} .
$$

The decimation factor in azimuth is given by:

$$
L=\left\lfloor\frac{\theta_{n \Delta}}{\theta_{\Delta}}\right\rfloor-1,
$$

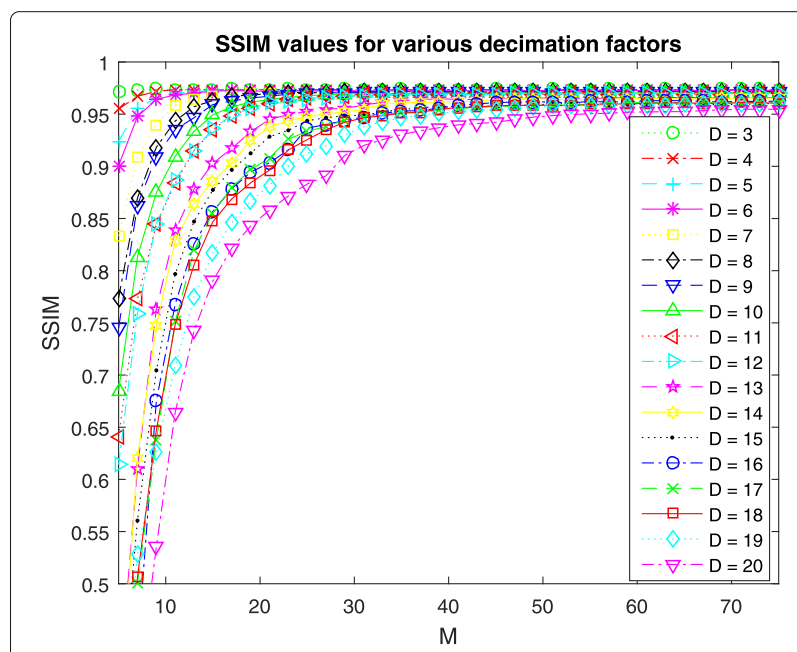

Fig. 10 SSIM results of "Washington D.C." $(1024 \times 1024)$ over various filter lengths and decimation factors 
and the decimated phase history used in back-projection of the digitally spotlit area is given by:

$$
\begin{aligned}
& X_{\text {alf }, i, j}[k, \theta]=F_{a}\left\{X_{r d, i, j}[k, \theta]\right\}, \\
& X_{s p, i, j}[k, \theta]=X_{\text {alf }, i, j}[k, L \theta] .
\end{aligned}
$$

$F_{a}\{\cdot\}$ is the low-pass filtering operation applied in the azimuth dimension, and $X_{s p, i, j}[\cdot]$ is the digitally spotlit VPH for region $(i, j)$. The digitally spotlit phase history can then be back-projected to form a subsegment of the original imaging scene, whose center is $P_{C}(i, j)$. The complexity of the back-projection process of $X_{s p, i, j}[\cdot]$ is significant reduction from the back-projection of $X_{p h}[\cdot]$ considering there are a factor of $D L$ fewer samples in $X_{s p, i, j}[\cdot]$, and those samples are projected onto an imaging plane which has a factor of $D^{2}$ fewer samples.

\section{Filter window results and discussion}

A number of windowing functions have been used in the literature for SAR processing. In [9], a Hamming window is used on the LPF for digital spotlighting. Additionally, subsequent articles have used Taylor windows and raised cosine windows in different SAR processing techniques $[10,11]$. In the following study, six different windows are compared: rectangular, Hamming, Blackman, Taylor $(\bar{n}=$ $5)$, raised cosine, and Kaiser $(\beta=5)$. The Blackman and Kaiser windows are included due to their powerful stopband suppression capabilities, and Taylor order $(\bar{n}=5)$ and Kaiser $(\beta=5)$ parameters are set because those particular variants performed the best over a number of values tested.

In the study, four images are considered: "Stockton," "San Francisco," "Pentagon," and "Washington, D.C." All of the images can be found at [12]. Each image is a $1024 \times 1024$ color image, and they are converted to grayscale. Additionally, each image is cropped from the center to form four $512 \times 512$ grayscale images. Each of these eight images is fed into the SAR simulator with digital spotlighting enabled to test different variables of the digital spotlight processing. The converted grayscale images are given in Fig. 4.
The simulator options are as follows: We define $P_{O}=$ $(0,0,0)$ and $P=(3696,1531,2800) \mathrm{m}$ at the center of the synthetic aperture. $R_{0}=707.1 \mathrm{~m}$. All radar parameters, number of pulses, and azimuth step size are determined by [13].

For image quality analysis, the Structured Similarity (SSIM) metric is used [7]. For filter window analysis, each of the windows is used over a range of filter orders on each of the test images. SSIM results are collected and analyzed over a range of $D \in[3,20]$. The filter length is $2 M+1$ where $M \in[5,75]$ is incremented in steps of 2 . Results for four of the images and different decimation factors are given in Fig. 5.

In Fig. 5, two conclusions can be made. First, all windowing methods eventually result in good overall digital spotlighting performance with the exception of the rectangular window. Regardless of filter order, the rectangular window performs below that of the other windowing methods. Second, the Taylor window $(\bar{n}=5)$ performs the best of the windows tested. Although only $D \in$ $\{6,8,14,18\}$ digital spotlighting segments are shown for a few images in Fig. 5, the Taylor window outperforms the others through the entire range of $D=[3,20]$ across all test images. We can visually see the effects of varying the type of filter window in digital spotlighting. Figure 6 gives a digitally spotlit "San Francisco" image with both rectangular and Taylor $(\bar{n}=5)$ windowing applied. A zoomed in section of Fig. 6 is provided in Fig. 7. In addition to synthetic VPH, the AFRL GOTCHA phase history from a 2006 data collect [14] is processed with the simulator to validate the digital spotlighting process. The results of digital spotlighting the GOTCHA dataset are given in Fig. 8, with a zoomed in section given in Fig. 9.

As shown in Figs. 6 and 7, when utilizing the rectangular window, aliased regions are prominent, and there are visual bordering effects at the intersection between spotlit regions. Conversely, when applying a Taylor $(\bar{n}=$ 5) window, these artifacts are significantly reduced and not readily visible. Additionally, we can see the improved image quality utilizing a Taylor window over a rectangular window in the GOTCHA data, provided in Figs. 8 and 9.

Table $1 M_{a s m}$ for various decimation factors and imagery

\begin{tabular}{lllllllllllllllllll}
\hline D & 3 & 4 & 5 & 6 & 7 & 8 & 9 & 10 & 11 & 12 & 13 & 14 & 15 & 16 & 17 & 18 & 19 & 20 \\
\hline "Stockton" $(512 \times 512)$ & 5 & 9 & 11 & 13 & 17 & 21 & 21 & 27 & 31 & 31 & 33 & 37 & 41 & 47 & 47 & 49 & 53 & 55 \\
"Stockton" $(1024 \times 1024)$ & 5 & 7 & 9 & 13 & 17 & 17 & 21 & 23 & 25 & 25 & 37 & 41 & 37 & 41 & 45 & 45 & 49 & 59 \\
"San Francisco" $(512 \times 512)$ & 7 & 11 & 11 & 13 & 19 & 23 & 25 & 29 & 31 & 33 & 39 & 39 & 41 & 55 & 57 & 53 & 55 & 67 \\
"San Francisco" $(1024 \times 1024)$ & 5 & 7 & 11 & 15 & 19 & 17 & 23 & 27 & 27 & 29 & 45 & 49 & 47 & 43 & 57 & 55 & 53 & 67 \\
"Pentagon" (512 × 512) & 7 & 9 & 11 & 11 & 19 & 21 & 21 & 27 & 31 & 31 & 33 & 35 & 41 & 45 & 45 & 49 & 51 & 57 \\
"Pentagon" (1024 × 1024) & 5 & 7 & 9 & 13 & 17 & 17 & 19 & 23 & 25 & 25 & 35 & 39 & 37 & 41 & 43 & 43 & 47 & 55 \\
"Washington, D. C." (512 $\times 512)$ & 7 & 9 & 11 & 11 & 15 & 21 & 19 & 25 & 29 & 29 & 31 & 35 & 41 & 39 & 47 & 51 & 51 & 55 \\
"Washington, D. C." $(1024 \times 1024)$ & 5 & 9 & 11 & 11 & 15 & 17 & 21 & 23 & 25 & 27 & 33 & 35 & 37 & 41 & 43 & 41 & 49 & 55 \\
\hline
\end{tabular}




\section{Filter order results and discussion}

An additional parameter for digital spotlighting is the filter order, or length of the FIR filter's impulse response in the decimation in range and azimuth. According to Kaiser's FIR-length approximation, the required filter order $M$ is linearly proportional to the digital spotlighting segments $D$. Figure 10 shows SSIM values of the "Washington D.C." image across several filter orders and several decimation factors and applying a Taylor $(\bar{n}=5)$ window to the filter coefficients.

In Fig. 10, it is readily shown that as the decimation factor increases, the required filter order $M$ increases as well. Careful analysis of these curves allow us to determine the minimum value of $M$ for each decimation factor which maximizes the SSIM value. This filter order is referred to as $M_{a s m} . M_{a s m}$ is defined as the minimum value of $M$ where the SSIM score is greater than or equal to 0.99 of the maximum SSIM score over the range of $M$ (i.e., asymptotically close to the maximum SSIM score). Table 1 gives $M_{\text {asm }}$ generated from all test images.

From the Kaiser FIR approximation, it is known that $M_{\text {asm }}$ linearly increases with increasing $D$. A linear fit is applied to the data in Table 1 to create a simple calculation for the required filter order for digital spotlighting. Fitting the data to a line, we obtain:

$$
\tilde{M}_{\text {asm }}[D]=\lfloor(s D+b)+0.5\rfloor, \quad D>1,
$$

where $s=2.95$, and $b=-4.15$. Figure 11 gives a visual representation of the data in Table 1 and calculated $\widetilde{M}_{\text {asm }}[\cdot]$.

As shown in Fig. 11, the values for $M_{\text {asm }}$ over a fairly wide range can be fairly well approximated with a linear fit for each of the images tested. Thus, the FIR filter order required for digital spotlighting in general may be estimated using Eq. 21.

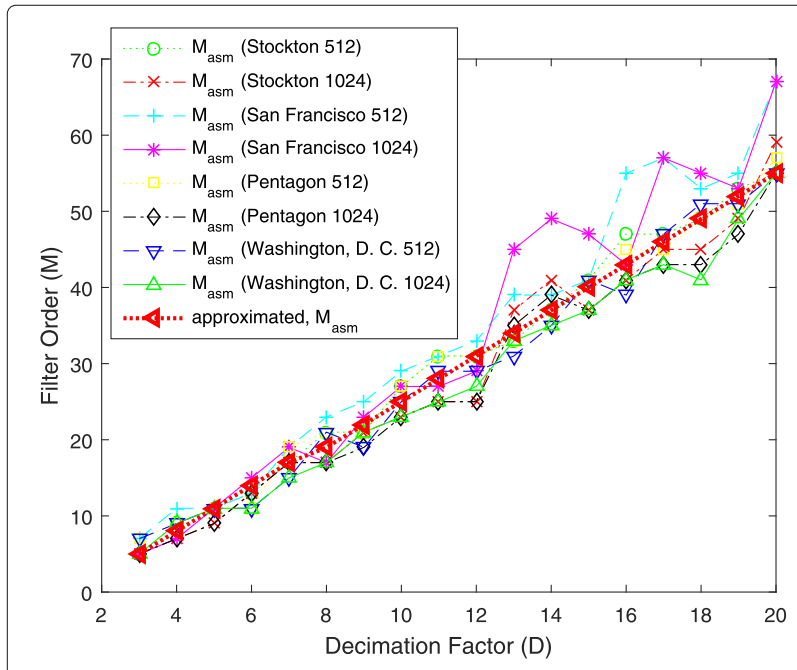

Fig. 11 Decimation factor $D$, verses filter length $M$, and $\widetilde{M}_{\text {asm }}[\cdot]$

\section{Conclusion}

This paper presents an objective evaluation of filter parameter selection for digital spotlighting in SAR imagery. The analysis is generated from a SAR image formation simulator which uses imagery to form the phase history of the radar. Because of this, the quality of the reconstructed SAR image may be objectively evaluated using well-known image processing metrics. From the analysis, it is shown that the Taylor window $(\bar{n}=5)$ provides the best image quality over a wide range of filter lengths and decimation factors. Additionally a simple linear relationship between the decimation factor and filter order is found.

\section{Abbreviations}

FIR: Finite impulse response; iFFT: Inverse Fourier transform; LFM: Linear frequency modulated; SAR: Synthetic aperture radar; VPH: Video phase history

\section{Acknowledgements}

The authors would like to thank the US Air Force Research Laboratory for funding this effort

\section{Authors' contributions}

The authors' contributions to digital spotlighting processing are as follows: We have created a SAR simulator which can objectively evaluate different parameter selection of the digital spotlighting process, namely filter order and filter type used in the decimation of the VPH. We have additionally found a simple linear relationship between appropriate filter order and decimation factor. SAR processing developers can use this relationship to easily calculate the proper filter order to use for digital spotlighting. All authors read and approved the final manuscript.

\section{Funding}

Funding of this work was provided by the US Air Force Research Laboratory, Sensors Directorate.

\section{Availability of data and materials}

Images used in this study can be found at [12]. Additionally, the GOTCHA VPH data is available at [14].

\section{Competing interests}

The authors declare that they have no competing interests.

\section{Author details}

${ }^{1}$ Department of Electrical and Computer Engineering, Kettering Laboratory, University of Dayton, Rm. 341, 300 College Park, Dayton, OH 45469, USA.

${ }^{2}$ University of Dayton Research Institute, 300 College Park, Dayton, OH 45469, USA. ${ }^{3}$ Sensors Directorate, United States Air Force Research Laboratory, Wright-Patterson AFB, Dayton, OH 5433, USA.

Received: 28 March 2018 Accepted: 9 September 2019

Published online: 15 November 2019

\section{References}

1. M. Soumekh, in Proceedings of Ist International Conference on Image Processing, vol. 1. Digital spotlighting and coherent subaperture image formation for stripmap synthetic aperture radar (IEEE, 1994), pp. 476-480

2. A. Dallinger, S. Schelkshorn, J. Detlefsen, Efficient $\omega$-k-algorithm for circular SAR and cylindrical reconstruction areas. Adv. Radio Sci. 4.B. 3, 85-91 (2006)

3. L. Nguyen, et al., Enhancement of backprojection SAR imagery using digital spotlighting preprocessing (IEEE, 2004)

4. M. Soumekh, et al., Signal processing of wide bandwidth and wide beamwidth P-3 SAR data. IEEE Trans. Aerospace Electr. Syst. 37(4), 1122-1141 (2001)

5. E. J. Balster, F. A. Scarpino, A. M. Kordik, K. L. Hill, Synthetic aperture radar imaging simulator for pulse envelope evaluation. J. Appl. Remote Sensing. 11(4), 046022 (2017) 
6. E. J. Balster, et al., in Proceedings of the 5th International Workshop on OpenCL. Gpgpu acceleration using opencl for a spotlight sar simulator. (ACM, 2017), p. 1

7. Z. Wang, et al., Image quality assessment: from error visibility to structural similarity. IEEE Trans. Image Process. 13(4), 600-612 (2004)

8. E. J. Balster, F. A. Scarpino, A. M. Kordik, K. L. Hill, Synthetic aperture radar imaging simulator for pulse envelope evaluation. J. Appl. Remote Sensing. 11(4), 046022 (2017)

9. K. E. Dungan, L. A. Gorham, L. J. Moore, in Algorithms for Synthetic Aperture Radar Imagery XX. Vol. 8746. SAR digital spotlight implementation in MATLAB (International Society for Optics and Photonics, 2013)

10. A. W. Doerry, Anatomy of a SAR impulse response. No. SAND2007-5042. (Sandia National Laboratories, 2007)

11. C. V. J. Jakowatz, et al., Spotlight-Mode Synthetic Aperture Radar: A Signal Processing Approach. (Springer Science \& Business Media, 2012)

12. USC Signal and Image Processing Institute. http://sipi.usc.edu/database. Accessed 31 July 2019

13. L. A. Gorham, L. J. Moore, in Algorithms for Synthetic Aperture Radar Imagery XVII. Vol. 7699. SAR image formation toolbox for MATLAB (International Society for Optics and Photonics, 2010)

14. GOTCHA 2008 Dataset. https://www.sdms.afrl.af.mil/index.php? collection=gotcha. Accessed 31 July 2019

\section{Publisher's Note}

Springer Nature remains neutral with regard to jurisdictional claims in published maps and institutional affiliations.

\section{Submit your manuscript to a SpringerOpen ${ }^{\circ}$ journal and benefit from:}

- Convenient online submission

- Rigorous peer review

- Open access: articles freely available online

- High visibility within the field

- Retaining the copyright to your article

Submit your next manuscript at $\gg$ springeropen.com 